\title{
Reversal learning as a function of length of the deprivation schedule and the amount of training*
}

\author{
DOUGLAS HARGRAVE, FRANK WOOD \\ and \\ CHARLES L. RICHMAN \\ Wake Forest University, Winston-Salem, N.C. 27109
}

In ORE studies, prior to reversal learning, overtrained rats are maintained on a food deprivation schedule for a longer period of time (the number of days in overtraining) than criterion-trained rats. This procedure results in a confounding between training and drive. The present research suggests that the overtrained animals' extended food deprivation schedule does not contribute to the ORE.

If overtrained animals reversal learn more rapidly than criterion-trained animals, the phenomenon is called the overtraining reversal effect (ORE). Typically, the procedure is to begin a food deprivation cycle at least 3 or 4 days prior to Day 1 of discrimination $\mathrm{T}$-maze training (e.g., white alley positive, black alley negative). Original learning criterion is usually set at a criterion of $90 \%$ correct responses in a single day's run. Upon reaching criterion, half of the animals are given reversal training (black alley positive, white alley negative); whereas the remaining animals are given $\mathrm{N}$ days of overtraining past criterion (e.g., 16 days) prior to reversal training. The resulting ORE is attributed to the facilitation of reversal learning through extended training. The hypothesis adopted here is that training has been confounded with drive. Since it has been shown that the initial learning rate in a simultaneous discrimination problem is related to drive (Richman, Gardner, Montgomery, \& Benewicz, 1970), it is possible that in overtrained animals, which have been maintained on a deprivation schedule a greater number of days than criterion-trained animals, the ORE is at least partially due to the overtrained Ss' relatively high level of drive prior to the onset of reversal training. The present study was conducted to parcel out the effects of extended training from time on a deprivation schedule in order to determine if one or both factors contribute to the ORE.

\section{METHOD}

\section{Subjects}

The Ss were 32 90-day-old, experimentally naive, male albino rats.

\footnotetext{
*A summary of this study was presented at the 1972 annual meeting of the Southeastern Psychological Association, Atlanta, Georgia. The research was supported in part by a grant from the Wake Forest University Research and Publications Fund and by Grant MH 16962-02 from the National Institute of Mental Health, U.S. Public Health Service. Reprints may be obtained from Charles L. Richman, Department of Psychology, Wake Forest University, Winston-Salem, North Carolina 27109.
}

\begin{abstract}
Apparatus
Two identically constructed steel-gray $\mathrm{T}$ mazes were used to test the animals. The alleys were $16 \mathrm{in}$. high and $5 \frac{1 / 2}{2}$ in. wide; the startboxes were $14 \mathrm{in}$. long, and the start stems and goal alleys were 10 and 18 in. long, respectively. Food pellet containers, inverted bottle tops, were located 1 in. from the end of the goalboxes. Guillotine doors were placed between the startboxes and start stems and between the start stems and the goal alleys. Four-inch-wide black and white inserts were located in the goal alleys, $1 \frac{112}{2}$. on either side of the center of the start stem. One of the maze's left goal alleys contained the black insert and the right alley, the white insert; whereas, the other maze's left goal alley contained the white insert and the right alley, the black one. The start stems of both mazes were oriented in the same direction (e.g., north to south).
\end{abstract}

\section{Procedure}

Beginning 4 days prior to Experimental Day 1, and throughout the experiment, all animals were weighed every third day and maintained on a 22-h food deprivation cycle and an ad lib water schedule.

Four groups of eight animals each (group mean body weights ranged from 313 to $315 \mathrm{~g}$ on Experimental Day 1) were trained to a seven out of eight original and reversal learning criterion: Group Criterion 1 (C1) Ss began discrimination training on Day 1; reversal training began on the day following these $\mathrm{Ss}^{2}$ obtainment of seven out of eight correct responses. Group Overtrained (OT) began original training on Day 1 also; however, upon reaching initial criterion, these animals were given 16 days of overtraining (eight trials per day). One Group OT animal died during overtraining, reducing the size of this group to seven Ss.

On Experimental Day 17, Groups Criterion 2 and 3 (Groups $\mathrm{C} 2$ and $\mathrm{C} 3$, respectively) began initial training and were trained to the seven out of eight initial criterion level, immediately followed by the onset of reversal learning. Time on deprivation for Groups C2 and C3, prior to reversal learning, was equal to that of Group OT Ss, but their training level was equal to that of the criterion-trained Ss. The only procedural differences employed between Groups $\mathrm{C} 2$ and $\mathrm{C} 3$ were that the latter animals were picked up eight times per day during the first 16 experimental days and were given 5 min maze exploration during the 2 days prior to Day 1 . This procedure insured that Ss in Groups C3 and OT were handled an equal number of times prior to reversal training. During initial training, half of the animals in each group were trained with the black (white) alley positive and white (black) alley negative. Reward magnitude was $3-100 \mathrm{mg}$ Noyes food pellets. Animals were retained in the negative goalbox for $15 \mathrm{sec}$. The trial by trial maze order was randomly determined by a Fellow's (1967) series.

\section{RESULTS AND DISCUSSION}

Table 1 shows the mean number of days to original and reversal learning criterion. Between-group differences were assessed with the Mann-Whitney U test. Since Groups $\mathrm{Cl}$ and OT were treated identically until they reached the criterion of seven out of eight correct responses, these $\mathrm{Ss}^{\prime}$ days to initial criterion were combined. Although initial learning differences were not found between Group C1-OT and Group C2 $(Z=1.26$, $p>.05)$, Group C3 animals reached criterion in fewer trials than Group C1-OT $(Z=2.07, p<.05)$. Since Group C2 and C3 mean body weights were 
Table 1

Mean Days to Criterion

\begin{tabular}{ccc}
\hline & $\begin{array}{c}\text { Initial } \\
\text { Learning }\end{array}$ & $\begin{array}{c}\text { Reversal } \\
\text { Learning }\end{array}$ \\
\hline Group OT & 6.14 & 3.57 \\
Group C1 & 6.62 & 6.13 \\
Group C2 & 5.00 & 5.50 \\
Group C3 & 4.13 & 5.50 \\
\hline
\end{tabular}

approximately the same at the completion of initial training $(22.7 \%$ and $24.6 \%$ mean body weight loss, respectively) but greater than those of Groups $\mathrm{C} 1$ and OT ( $15.4 \%$ and $16.5 \%$ weight loss, respectively), these results suggest that the interaction of high drive and daily handling of Group C3 animals prior to discrimination training had an appreciable affect on the rate of learning.

Days to reversal learning criterion was of prime concern in the present study (Table 1). If it was found that Ss in Groups C1 and C2 (C3) took an equal number of days to reach the reversal learning criterion but their performances were retarded relative to Group OT, then it could be argued that the ORE was solely a function of extended training. If, however, Group OT and Group C2 (C3) reversal learning scores did not differ but these animals took significantly fewer days to reach reversal criterion than Group C1, then the ORE could be attributed to the extended deprivation schedule. And, finally, if Group C1 Ss' reversal learning rate was retarded relative to Group OT, i.e., the ORE, but Group C2 (C3) reversal learning scores were not significantly different from either Group C1 or
Group OT, then the ORE could be interpreted as being a function of the interactive effects of extended training and food deprivation.

A Kruskal-Wallis one-way analysis of variance indicated that reversal learning differences were present between groups $(\mathrm{H}=7.30, \mathrm{df}=3, \mathrm{p}<.055)$. The ORE was demonstrated; Ss in Group OT took significantly fewer days to reach the reversal learning criterion than those in Group $\mathrm{C} 1(\mathrm{U}=10.5, \mathrm{p}<.020)$. Furthermore, the Group $\mathrm{C} 1$ animals' reversal learning scores were not different $(p>.20)$ from those in Group C2 (C3). Last, and most important, Group OT Ss' rate of reversal learning was more rapid than those in Groups $\mathrm{C} 2$ and $\mathrm{C} 3$ $(U=11.00, p<.027$ and $U=7.5, p<.010$, respectively). These results indicate that ORE, as suggested by traditional researchers (Reid, 1953; Mackintosh, 1969; etc.), is due solely to the effects of extended training and is not a function of the length of the deprivation schedule prior to the onset of reversal learning.

\section{REFERENCES}

Fellows, B. J. Chance stimulus sequence for discrimination tasks. Psychological Bulletin, 1967, 2, 87-92.

Mackintosh, N. J. Further analysis of the overtraining reversal effect. Journal of Comparative \& Physiological Psychology, $1969,67,1-18$.

Reid, L. S. The development of noncontinuity behavior through continuity learning. Journal of Experimental Psychology, $1953,46,107-112$.

Richman, C. L. Gardner, J. T., Jr., Montgomery, M., \& Benewicz, K. Effects of body weight on position and brightness discrimination tasks. Learning \& Motivation, 1970, 1, 218-225.

(Received for publication October 5, 1972.) 